Straßburg 1881. - Eisler, P., Die Muskeln des Stammes, 1912. - Eppinger, Allgemeine und spezielle Pathologie des Zwerchfells. Nothnagel, Spezielle Pathologie und Therapie, Suppl. I, 1911. - Gaillard, De la hernie diaphragm. d'origine congénitale, Thèse de Paris 1907. - Gautier, Contribution à l'étude de la hernie diaphr. cong. Thèse de Paris 1897. - v. Gößnitz, Eìn weiterer Beitrag zur Morphologie des Zwerchfells. Jenaische Ztschr. f. Naturwissensch, Bd. 39, 1905, S. 235-244. Desgl. Bd. 38, S. 619-672. — G r o s ser, Über Zwerchfellshernien. Wien. klin. Wschr. 1899, S. 655-657. - Haberlah, Vergleichende Untersuchungen über den Bau des Zwerchfells der Haussähgetiere. Dresden 1911. - Heller, Diskussion zum Vortrag Benekes: "Über Bauchlunge u. Hern. diaphr. spuria." Verh. d. Path. Ges. Bd. 9, Meran 1905, S. 210. - His, W., Anatomie menschlicher Embryonen, 1880, Bd. 1. - Derselbe, Mitteilungen zur Embryologie des Menschen und der Säugetiere. Arch. f. Anat. u. Physiol. 1881. - Hoffmann, G., Über Zwerchfellbrüche. I.-D. Breslau 1905. - Jourdan, Art. Hépatocèle. Dict. des sciences méd. Paris 1817. - Kantor, Zwei Fälle von Lebermißbildung. Virchows Arch., Bd. 174, 1903, S. 571-576. - Keck, Zur Kenntnis der Zwerchfellshernien bei Neugeborenen und ihrer Entwicklung. Wien. klin. Rundsch., Bd. 25, 1911, S. 789, 806. - Keibel u. Elze, Normentafeln zur Entwicklungsgeschichte der Wirbeltiere, 1908. - Keibel u. Mall, Handbuch der Entwicklungsgeschichte des Menschen, Bd. 1, 1910, S. $534 \mathrm{ff}$ - Keith, The nature of the mammalian Diaphragme and Pleural-Cavities. Journ. of anat. u. phys., vol. 39, 1905, S. 243. - Klebs, Hernia diaphragmatica dextra mit Fraktur des rechten Rippenknorpelrandes und grauer Degeneration des Rückenmarks. Virchows Arch. Bd. 33, 1865, S. 445-447. - Koch, Der Zwerchfellbruch. Münch. med. Wschr., Bd. 55, 1908, S. 1431-1433. - Kohn, Über Hernia diaphragm. congenita. I.-D. Erlangen 1874. - Kollmann, J., Lehrbuch der Entwickhungsgeschichte des Menschen, 1898, S. 298-300. - Leichtenstern, in v. Ziemssen: Handbuch der speziellen Pathologie u. Therapie, Bd. 7, 2. Hälfte, 1878, S. 460-468. - Liepmann, W., Zur Åtiologie der kongenitalen Zwerchfellshernien. Arch. f. Gyn., Bd. 68, 1903, S. 780-800. - Lockwood, The early development of the pericardium diaphragme and the grand veins. Philos. Transact. of the Royal acad. of London, vol. 179, 1888. - Luksch, Echte erworbene Zwerchfellshernie. Prag. med. Wschr., Bd. 28, 1903, S. 169-171. - Mazilier, Contrib. à l'étude de l'embryologie du diaphragme. Thèse de Paris 1907. - Mayer, 0., Über Hernia diaphragm. congenita. I.-D. Berlin 1891. - Pape, C. E., Über Hernia diaphragm. vera mit einem durch die Leberanlage gebildeten Bruchsack. I.-D. Leipzig 1904. - Schwalbe, E., Beobachtung eines Falles von Hernia diaphragm. vera. Ztbl. f. allg. Path. u. path. Anat., Bd. 11, 1900, S. 262. - Derselbe, Die Morphologie der Mißbildungen des Menschen und der Tiere, 1906, Teil I, S. 146. - Swaen, Recherches sur le développement du foie, du tube digestif, de l'arrière cavité du péritoine et du mésentère. Journ. de l'anat. et de la phys. 1897. - Uskow, N., Über die Entwicklung des Zwerchfells, des Perikardiums und des Zökums. Arch. f. milrros. Anat., Bd. 22, Boun 1883, s. 143 bis 219. - Waldeyer, Über die Beziehungen der Hernia diaphragm. congenita. Zur Entwicklungsweise des Zwerchfells. Breslau 1884. - Winkler, H., Eine angeborene Zwerchfellshernie mit bemerkenswerten Mesenterialverhältnissen und anderen Besonderheiten. Frankf. Ztsehr. f. Path. Bd. 6, 1911, H. 3. - Zurhelle, Ein Beitrag zur Lehre von der Entstehung der Zwerchfellsbrüche. I.-D. Bonn 1904.

\title{
VI.
}

\section{Beiträge zur Lehre vom kongenitalen Zwerchfellsdefekt mit besonderer Berücksichtigung des rechtsgelegenen.}

(Aus dem Pathologisehen Institut der Kaiser Wilhelms-Universität zu Straßburg i. E.)

Von

Priv.-Doz. Dr. Georg B. Gruber,

II. Assistent am Institut.

(Hierzu 13 Textfiguren.)

Bei dem gewiß nicht so seltenen Vorkommen von angeborener Lückenbildung im Zwerchfelle mag es vielleicht überflüssig erscheinen, weitere Mitteilungen zu 
diesem Thema zu machen. Sind doch in der Literatur (Eppinger, Cailloud) ${ }^{\mathbf{1}}$ ) unter der Benennung „falsche Zwerchfellshernien" etwa 250 Einzelfälle bekannt, ungeachtet der vielen Fälle, welche von der Veterinärmedizin registriert worden sind (v. Gößnitz [Literatur!], Stöber, Joest). Aus dieser Fülle von Mitteilungen kann jedoch nicht geschlossen werden, daß das vorliegende Gebiet geklärt ist. Noch heute gibt uns jede neue Beobachtung von kongenitaler Zwerchfellsmißbildung Rätsel auf, Rätsel in verschiedener Hinsicht, solche, welche sich auf die Zwerchfellsentwicklung beziehen, und solche, welche die Entwicklung und Topographie der Bauch- und Brusteingeweide ins Auge fassen; damit rollen sich stets wieder die Fragen nach dem Zeitpunkt und der Ursache solcher Lückenbildungen auf, ferner die Fragen nach den Folgen, die der mehr oder weniger große Zwerchfellsdefekt nach sich zieht. Bedenkt man außerdem den auffälligen Unterschied in der Topographie der Zwerchfellsdefekte, die Häufigkeit, mit der sie links, die Seltenheit, mit der sie rechts gefunden worden sind, so ist vielleicht doch die Mitteilung von einschlägigen Fällen unter Berücksichtigung all dieser Fragen nicht ungerechtfertigt. Die Beobachtungen, welche meinen Ausführungen zugrunde liegen, konnten an einer Serie von 12 Präparaten des hiesigen pathologisch-anatomischen Museums gemacht werden ${ }^{1}$ ). (Meinem Chef, Herrn Professor H. Chi a ri, danke ich für die Überlassung des Materials bestens.)

Es handelt sich um vier Beobachtungen von Zwerehfellsdefekt auf der rechten Seite und um 8 Fälle der linken Seite.

\section{A. Rechtsseitige Zwerchfellslücken.}

Fall 1. (Mus.-Katalog Nr. 3824.) 9 Tage alter Knabe aus der Franenklinik. (Geheimrat Prof. Dr. Fehling.) In dem am 28. März 1904 aufgenommenen Sektionsprotokoll heißt es: Gut entwickeltes Kind, ziemlich starke Zyanose des Gesichtes. Aus dem Nabelstumpf entleert sich auf Druck etwas Blut. Nabelarterien weit offen. Im Nabelbereich keine Anzeichen von Entzündung. In der Bauchhöhle etwa $2 \mathrm{ccm}$ seröser Flüssigkeit. Der rechte Leberlappen liegt nur in sehr kleiner Ausdehnung zutage. Er erstreckt sich mit seiner oberen Hälfte durch ein Loch in der rechten Zwerchfellshälfte in den Brustraum. Auch die Gallenblase, wie der untere Leberrand sind in die rechte Pleurahöhle verlagert. Beim Herausholen des Leberlappens entleert sich aus der Pleurahöhle eine Menge von etwa $30 \mathrm{ccm}$ klaṛer seröser Flüssigkeit. Der Ductus choledochus bildet einen Bogen zum Duodenum hin, er ist durchgängig. Die rechte Lunge ist sehr klein, namentlich ihr Unterlappen sehr atelektatisch; Auch die oberen Teile dieser Lunge sind fast gänzlich luftleer. Das Herz ist groß, beide Vorhöfe erscheinen prall gefüllt. Das Foramen ovale ist größtenteils durch eine Membran geschlossen, jedoch im oberen Teil breit durchlöchert. Der rechte Ventrikel etwas vergrößert, seine Wandung leicht verstärkt, der Ductus Botalli weit offen. In der lufthaltigen linken Lunge zahlreiche Ekchymosen, jedoch keine Verdichtungsherde. Die Milz ist dunkel, blutreich und etwas vergrößert.

Die Besichtigung des Präparates im Musenm läßt eine reichlich große Leber erkennen. Der

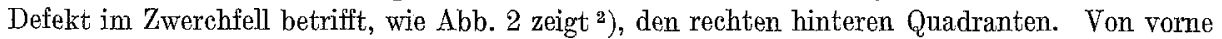
her ist bis zur elften Rippe eine muskuläre sichelförmige Zwerchfellspartie stehen geblieben. Auch. hinten zieht sich ein muskuläres Band unter der Serosa, welche an der Übergangszone von Brust-

1) Vgl. vorstehende Abhandlung.

2) Dazu dient als Vergleich Abbildung 1. 
und Bauchhöhle liegt, von der lumbalen Wirbelsäule gegen die zwölfte Rippe hin, ohne aber etwa dadurch eine Leiste oder Falte an der rückwärtigen Begrenzung der Lücke vortreten zu lassen; vielmehr ist die hintere Begrenzung der Zwerchfellslücke von der Thoraxwandung gebildet.

Die eben beschriebene Muskelleiste ist in dieser Form beim gewöhnlich ausgebildeten Zwerchfell nicht vorhanden, wohl aber ist sie als Variation bekannt und nach Eislers Ausführungen bereits von Albinus abgebildet worden. Eisler sagt darüber (S. 555): „Beim Fehlen einer Quadratusarkade breiten sich die vom zweiten Lendenquerfortsatze entspringenden Bündel des Crus laterale häufig so stark fächerförmig aus, daß sie nur zum kleinsten Teile noch das Zentrum tendineum erreichen. Vielmehr strahlen die Bündel der Reihe nach sehnig in die Faszie auf der thorakalen Fläche der Pars costalis ans, bis ein lateralster Bündelkomplex in flachem Bogen die 11. oder 12. Rippe dorsal und medial neben der dort entspringenden kostalen Portion erreicht...

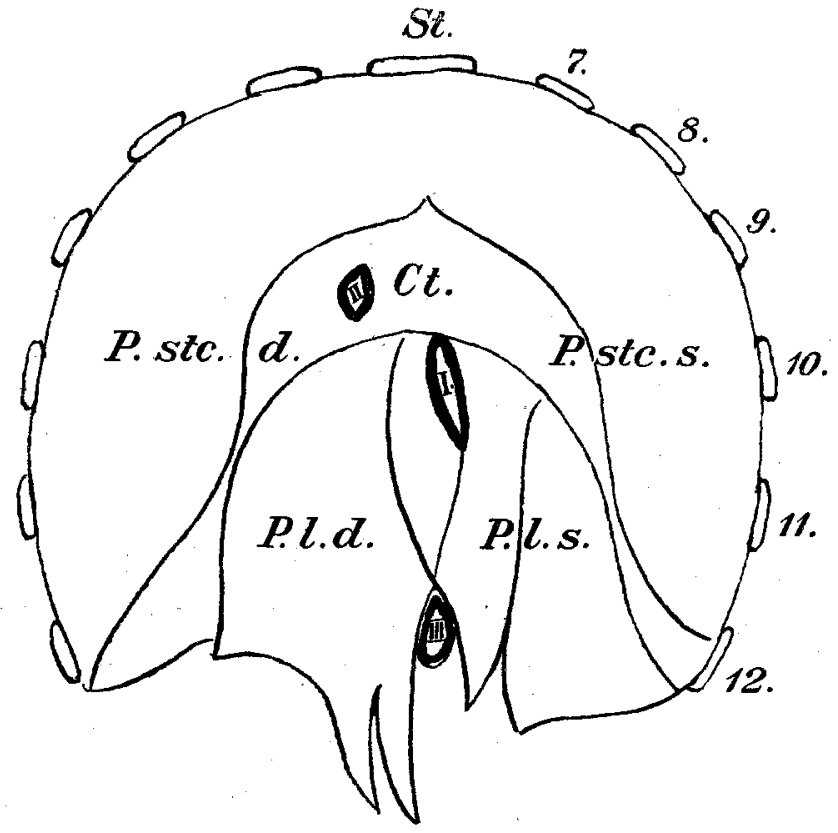

Fig. 1. Normalscbema des Zwerchfells (von unten gesehen) nach W. v. Gößnitz. $\mathrm{I}=$ Foramen oesophag. $\quad$ II $=$ Foramen quadrilat. III $=$ Hiatus aorticus $. \quad \mathrm{St}=\mathrm{Sternum}$. $\mathrm{Ct}=$ Centrum tendjneum. P. stc. d. = Pars sternocostalis dextra. P. ste. s. = Pars sterno costalis sin. P. l. d. = Pars lumbalis dextra. P. 1. s. = Pars lumbalis sin. 7.-12. Rippe.

Die Bündel dieser Portion können sich mit letzten Bündeln der kostalen Portion von der 12. Rippe durchflechten." Die Albinische Muskelleiste scheint bei Zwerchfellsdefekten öfter vorzukommen, wie einige weitere Fälle lehren.

Fall II. (Mus.-Kat. Nr. 2393.) Es handelt sich um ein seit langer Zeit im Museum aufbewahrtes Präparat, über dessen Herkunft nichts Näheres bekannt ist, und das nur einen Torso darstellt. Es stammt offenbar von einem neugeborenen Kind mit einer Hyperplasie der Thymus. Von der rechten Zwerchfellshälfte ist nur ventral ein sichelförmiger Rest erhalten (Abb. 3), der sich bis zur 10. Rippe hin erstreckt. Dorsal sieht man, wie im vorigen Falle, einen Streifen Muskulatur von der Wirbelsäule her im Bogen unter der nicht leistenförmig vorgewölbten Serosa im Bereiche des Pleura-Peritonäalübergangs bzw. von dem Hiatus oesophageus her gegen die 12. bzw. 11. Rippe hinziehen. Der Nervus phrenicus der reehten Seite teilt sich in zwei Äste, einen für die vordere Sichel und einen für die kleine dorsale Partie der Zwerchfellshälfte. Die sehr große 
Leber ist mit der Gallenblase dureh den Defekt in den Pleuraraum so weit ausgetreten, daß nur mehr der linke Lappen in der Bauchhöhle angetroffen wird. Der Darm zeigt ein Mesenterium commune, das nur in der Gegend der rechten Kolonflexur etwas angeheftet ist. Das Zökum liegt rückwärts von der Leber nach oben in der Zwerchfellslücke frei eingelagert, der Wurmfortsatz ist unter das Ileum geschlagen, das Colon transversum zieht wie gewöhnlich vor dem Duodenum vorbei.

In der Brusthöhle fällt eine starke Verdrängung des Herzens nach links auf. Zwischen Speiseröhre und Aorta ist das hintere Mediastinum etwas ausgedehnt, insofern hier die in die Brusthöhle vorgefallenen Baucheingeweide nach der linken Seite hingedrängt sind und eine Art Nische ausgestülpt haben. Die rechte Lunge ist nur andertungsweise dreilappig und scheint ebenso wie die linke vollkommen luftleer gewesen zu sein.

Fall III. (Mus.-Kat. Nr. 7756.) Es handelt sich um den von Cailloud ausfïhrlich geschilderten Fall eines 48jährigen Mannes (S.-Nr. 764, 17. September 1913), der an einer Hämorrhagie aus der Aorta infolge von Peri-Ösophagitis nach Fremdkörperverletzung der Speiseröhre gestorben war. Der Zwerchfellsdefekt dieses Mannes stellt einen Zufallsbefund bei der Obduktion dar. Im Leben waren zwar Anhaltspunkte für sein Bestehen bemerkt worden, aber sie konnten damals nicht entsprechend gedeutet werden, gewiß ein sehr entschuldbares Vorkommnis, nachdem

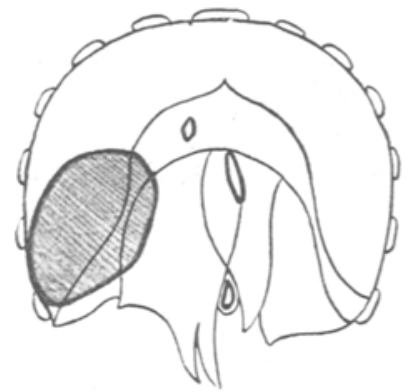

Fig. 2. Mus.-Kat. Nr. 3824.

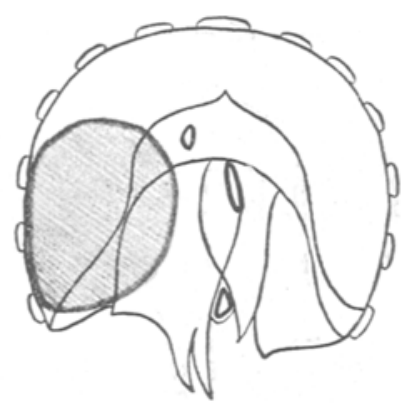

Fig. 3. Mus.-Kat. Nr. 2393.

der Patient bei der Sektion auch Zeichen einer rechtsseitigen Lungenspitzentuberkulose und partielle Pleuraverwachsungen darbot. Der Zwerchfellsdefekt sa $B$ hier im Gegensatz zu den vorher mitgeteilten Befunden nicht am Rande des Zwerchfells, sondern mehr zentral, immerhin aber näher der hinteren als der vorderen Thoraxwand, dort wo das Centrum tendineum an die Pars lumbocostalis angrenzt (Abb. 4). In seinex ganzen Umgebung war die Muskulatur wohl ausgebildet, und ganz unabhängig von Zwerchfellsdefekt lieb sich das Trigonum lumbocostale (Bochdaleksche Lücke) auspräparieren, das also von der Defektbildung völlig abgetrennt war. Der Rand des Defektes erschien allseitig dünn und gleichmäßig, nirgends mit den vorgefallenen Organen verwachsen. Der größte Teil der Leber war mit der Gallenblase in die Pleurahöhle vor gefallen; ferner war an der ventralen Begrenzung eine vom Colon transversum gebildete Schlinge in den Brustraum eingetreten. Die rechte Lunge war klein, hinten reichte sie entsprechend ihrem Ligamentum latum weit nach unten, sie hatte eine schiefe, nicht gleichmäßig ausgebildete kuppelförmige Basis, ihre drei Lappen waren gut zu erkennen. Eine Atelektase bestand nicht ${ }^{1}$ ).

Fall IV. (Mus.-Kat. Nr. 759.) Es handelt sich ebenfalls um ein von einem Erwachsenen stammendes Präparat, von dem leider nur das Zwerchfell und der daran haftende obere und rückwärtige Teil des rechten Leberlappens ohne alle Angaben über die Herkunft aufbewahrt worden sind. Das mit kräftiger Muskulatur versehene Zwerchfell zeigt etwas mehr ventral als im vorigen Faile, aber immer noch $3 \mathrm{~cm}$ von der linken vorderen Thoraxwand entfernt, eine nahezu kreis-

1) Dieser Fall wurde von mir im Unterels. Ärzte-Ver. am 20. Dez. 1913 demonstriert (Straßburger Med. Zeit. 1914, Nr. 1). 
förmige Lücke mit einem von unten her gesehenen, sehr gleichmäßigen, glatten Rande (Abb. 5). Auf der pleuralen Fläche ist der Rand stellenweise ctwas verdickt durch die gegen den Rand eingewachsene Muskulatur. Nirgends ist der Rand der Lücke mit der Leber verwachsen, von welcher sich entsprechend der Defektbildung ein kleiner Teil des rechten Lappens pilzartig und flach gegen die rechte Brusthöhle vorgewölbt hat. Das. Zwerchfell bildet zwischen der pilzartigen Vorragung und dem Hauptteil des rechten Leberlappens eine taillenartige Einschnürung. Der Durchmesser dieses Schnürringes bzw. des Zwerchfelldefeltes beträgt $8 \mathrm{~cm}$. Die Leber erhebt sich bis zu $3 \mathrm{~cm}$ über das Niveau des Defektes in die Pleurahöhle hinein. Mit Leichtigkeit kann man sie ans der Lücke des Diaphragma wie einen Knopf aùs dem Knopfloch entfernen.

\section{B. Linksseitige Zwerchfellslüeken.}

Fall I. (Mus.-Kat. Nr. 4385.) In der Franenklinik (Geheimrat Prof. Dr. Fehling) totgeborenes Mädchen, das äußerlich durch eine Myeloschisis lumbosacralis, wie durch einen linksseitigen Klumpfuß mißgebildet ist. Bei der durch Herrn Prof. v. Recklinghausen am 3. September 1903 vorgenommenen Sektion wurde festgestellt, daß in der Bauchhöhle nahezu der ganze

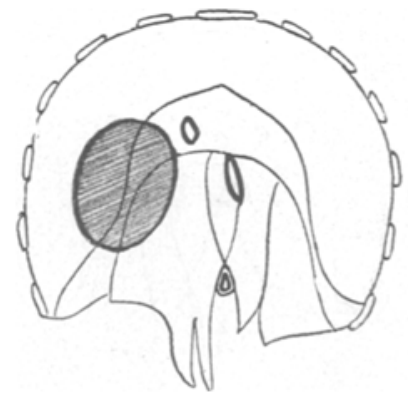

Fig. 4. Mus.-Kat. Nr. 7756.

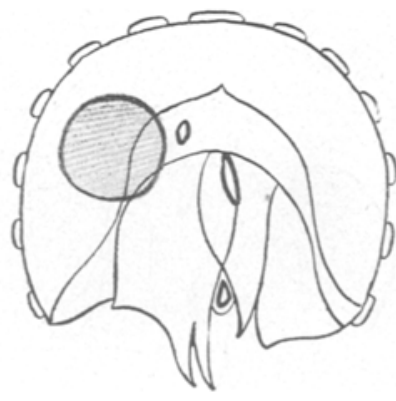

Fig. 5. Mus.-Kat. Nr. 759.

Darm nicht vorhanden war. Nur der Pylorus, das anschließende Duodenum, sowie der untere Teil des Colon descendens konnten im Abdomen festgestellt werden. Das Colon descendens war in der Mittellinie aufgehängt und adhärent, während im übrigen das ganze Darmgekröse frei war. Der fehlende Teil des Digestionstraktes ließ sich durch eine links im Zwerchfell gelegene Lücke in die Brusthöhle hinein verfolgen. Der Thorax erwies sich stark aufgetrieben, und zwar links wie rechts. Die linke Zwerehfellshälfte war nach unten konvex vorgebancht. Die Nieren erschienen groß und reichten weit nach unten. Auch die Leber war auffallend groß. Die Lüicke im Zwerehfell ließ neben dem Digestionstraktus noch leicht zwei Finger in die linke Brusthöhle einführen. An dem aufbewahrten Präparate fällt zunächst eine starke Verdrängung des Herzens nach rechts auf. Die Thymus entspricht den gewöhnlichen Verhältnissen, während die Schilddrüse die normale Größe überschritten hat. In der linken Pleurahöhle liegt gegen das hintere Mediastinum gebettet die Milz, ihr folgt nach vorne und etwas nach der Seite hin der Magen, das eingerollte Netz, das Kolon mit Blinddarm and Wurmfortsatz und der Dündarm. Das in der Bauchhöhle sichtbare Stück des Duodenum läßt am Präparat deutlich die Einmündung des Ductus choledochus erkennen. Vom linken Ligamentum triangulare hepatis sieht man ein kleines, zungenförmiges Gebilde nach oben in die Zwerchfellslücke hinein gebogen und aufwärts umgeschlagen, völlig plattgedrückt, etwa $6 \mathrm{~mm}$ groß und nur durch einen dünnen Stiel mit der Leber verbunden, in der Farbe völlig dem Lebergewebe gleichend. Die Insertion des Stieles befindet sich zunächst dem vorderen Rande der Zwerchfellslücke. Diese Lücke ist rückwärts und lateral gelegen (Abb. 6); ihre vordere Begrenzung wird gebildet von einer breiten Sichel der kostalen, muskulären Zwerchfellspartie, welche seitlich ihre Fasern bis über die 11. Rippe 
hinaus sendet. Medial und hinten zieht links von der Gegend des Ösophagus dorsal- und abwärts ein schmaler Muskelwulst, entsprechend dem Crus mediale des lumbalen Zwerchfellteiles. (Die mikroskopische Untersuchung des kleinen, zungenförmigen Leberläppchens am Rande der Zwerchfellslücke ergab innerhalo eines reichlichen Bindegewebsgerüstes offenbar in Wucherung begriffene Gallengänge, während von dem erdrückten Leberparenchym nur einige nekrotische und verkalkte Zonen bemerkt werden konnten.)

Fall II. (Mus.-Kat. Nr. 279.) Dieses Präparat betrifft einen weiblichen Fötus, der bereits in der französischen Zeit der medizinischen Fakultät zur Beobachtung gekommen war. Im Kataloge Ehrmanns ist er folgendermaßen benannt (Nr. 2862): Foetus à terme, auquel manque la portion ganche du diaphragme. Das stark gebleichte Präparat läßt im linken hinteren Quadranten des Zwerchfells eine Lücke erkennen, die groß genug ist, um den Daumen eines erwachsenen Menschen durchzalassen (Abb. 7). Vorne und seitlich wird diese Läcke von einer sichelförmigen Zwerchfellsfalte begrenzt, die sich rückwärts bis zum 11. Zwischenrippenraum, ja bis zur 12. Rippe hin erstreckt. Die hintere Begrenzung scheint bei oberflächlicher Besichtigung in gewöhnlicher Weise die Thorax-Abdominalwand zu bilden. Bei näherem Zusehen erkenntman jedoch deutJich,

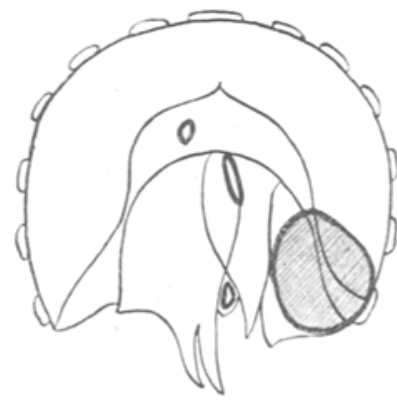

Fig. 6. Mus.-Kat. Nr. 4385.

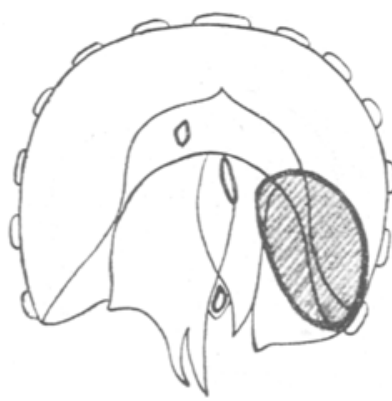

Fig. 7. Mus.-Kat. Nr. 279

was sich dann auch präparatorisch vollkommen zur Darstellung bringen läßt, daß unter der glatten und nicht leistenförmig vorspringenden Serosạ der hinteren Leibeshöhlenwand an Stelle des Crus laterale des lumbalen Zwerchfellteiles ein schmales Muskelband, über der Nebenniere quer verlaufend, sich aus der Gegend des Hiatus aorticus bzw. von der Wirbelsäule her bis zur 12. Rippe hinzieht, so daß der Defekt des Zwerchfells gewissermaßen vollkommen von Muskulatur umschlossen erscheint. (Dieses Verhalten entspricht dem Befunde in den Fällen I und II der rechten Seite.) Durch die Zwerchfellslücke sind Milz, Magen, Duodenum und ein Teil des linken Leberlappens in die linke Brusthöhle durchgetreten. Die Milz hat sich am weitesten an das Mediastinum angelagert, so daß dieses nischenartig zwischen Ösophagus und Aorta nach rechts ausgebaucht erseheint. Dort ist die Milz mit ihrem oberen Pol mit der Serosa des hinteren Mediastinums leicht verwachsen. Auch das Herz ist nach rechts verdrängt. Die linke Lunge ist zweilappig, sehr klein, die rechte Lunge verhält sich gewöhnlich. Die Nervi phreniei zeigen normalen Verlauf und teilen sich beiderseits auf dem Zwerchfell in einen dorsal und einen mehr seitlich ventral verlaufenden Ast. Der Herzbeutel wird von dem sehr großen, zweilappigen Thymus bedeckt. Von den Bauchorganen fällt die Leber durch ihre Größe auf. Ihr linker Lappen ist zum Teil scharnierartig durch die Zwerchfellslücke nach der Pleurahöhle umgeschlagen. Das Kolon besitzt ein freies Gekröse.

Fall III. (Mus.-Kat. Nr. 6638). Es handelt sich um einen am 19. August 1909 sezierten, $50 \mathrm{~cm}$ langen, $2485 \mathrm{~g}$ sehweren, bereits in Mazeration begriffenen männlichen Fötus aus der Frauenklinik (Geheimrat Prof. Dr. Fehling). Bei der Sektion ergab sich in beiden Pleurahöhlen und in der Bauchhöhle etwas sanguinolente Flüssigkeit. Die linke Pleurahöhle war größtenteils von einem Konvolut von Darmschlingen eingęnommen, welche durch eine ôffnung im linken Teil des 
Zwerchfells in die Pleurahöhle eingetreten waren und die zweilappige, aber sehr kleine luftleere linke Lunge völlig verdeckt hatten. Außer dem Dick- und Dünndarm, die sich durch ein freies Gekröse auszeichneten, waren noch die Milz, teilweise der Magen, ferner das Pankreas und das Netz vorgelagert. Die Milz hatte sich am weitesten hinten und medial so gegen das Mediastinum angedrängt, daß eine spaltförmige Nische zwischen Aorta und Speiseröhre durch Ausbauchung des Mittelfells nach rechts gebildet wurde. Die Leber zeichnete sich durch bedeutende Vergrößerung aus, während die Milz gewöhnliche Dimensionen aufwies. Der Zwerchfellsdefekt läßt sich an dem konservierten Präparat für drei Finger durchgängig erweisen. Er nimmt reichlich die Hälfte der linken Zwerchfellshälfte ein und ist vorne bis zur 11. Rippe hin von einer sichelartigen Zwerehfellsfalte begrenzt. Hinten erstreckt sich der Defekt bis zur Serosa der Körperwandung (Abb. 8). Eine eingehendere präparatorische Untersuchung konnte im Interesse der Intaktheit des Musealpräparates nicht vorgenommen werden.

Fall IV. (Mus.-Kat. Nr. 4453.) Torso eines nicht näher bezeichneten, im Jahre 1906 neugeborenen Mädchens mit einem partiellen, linksseitigen Zwerchfellsdefekt (Abb. 9), der sich nach rückwärts bis an die Körperwand erstreckt, während er vorn und seitlich von einer Zwerch-

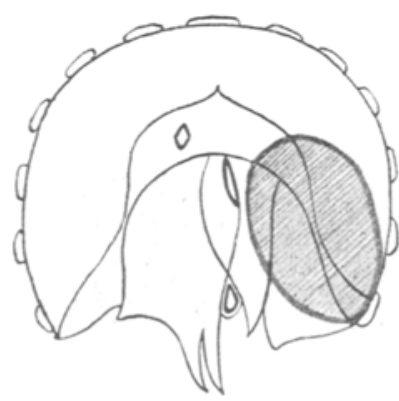

Fig. 8. Mus.-Kat. Nr. 6638.

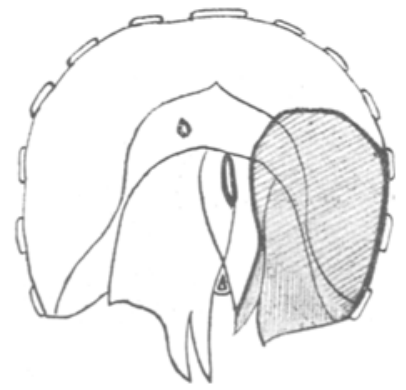

Fig. 9. Mus.-Kat. Nr. 4453.

fellsfalte sichelförmig bis zur 10. Rippe begrenzt ist. Durch diese Zwerchfellslücke ist in die linke Pleurahöhle vorgefallen: die am weitesten hinten und medial liegende Milz, welche das hintere Mediastinum Jeicht nach rechts vorgebaucht hat, das Zökum, das Colon transversum und der Anfangsteil des Colon descendens, Darmabschnitte, die an einem freien Gekröse befestigt sind. Ferner ist in die Brusthöhle eingetreten ein fast völlig abgeschnürter, scharnierartig nach oben aufgeschlagener Teil des linken Leberlappens. Die sehr kleine, nach oben hingedrängte linke Lunge ist zweilappig die rechte Lunge dreilappig. Das Herz ist ein wenig nach rechts verlagert.

Fall V. (Mus.-Kat. Nr. 4452.) Rumpf und Eingeweide eines am 11. Juni 1905 sezierten totgeborenen Knaben von der Hebammenschule des Herrn Prof. Dr. Freund. Abgesehen von einem linksseitigen partiellen Zwerchfellsdefekt zeigte die Leiche das Vorhandensein einer sog. Nebenlunge, die auf der linken Seite als polypöser Körper an einem dünnen Stiele in der Axillarlinie zwischen der 9. und 10. Rippe befestigt war und in einer Länge von 2,7 cm frei in die Pleurahöhle vorragte. (Die näheren Verhältnisse hinsichtlich der Nebenlungenbildung wurden bereits anderweitig von mir mitgeteilt.) Der Zwerchfellsdefekt ist für vier Finger durchgängig, er liegt seitlich und hinten. Vorne bildet sein Rand eine sichelförmige Zwerchfellsfalte, die sich bis zur 10. Rippe erstreckt. Hinten wird durch die von glatter Serosa überzogene Leibeshöhlenwand der Defekt begrenzt (Abb. 10). Durch die Zwerchfellslücke waren in die linke Pleurahöhle durchgetreten: die Milz, welche auch wieder in einer nach rechts vorgebauchten Nische des Mediastinums zwischen Ösophagus und Aorta lag, der Magen, das Pankreas, das Netz, ferner der ganze Darm mit Ausnahme des Duodenums und des Colon descendens. Der Darm hing an einem freien Gekröse. Da der Magen mit dem Pylorus an seiner gewöhnlichen Stelle fixiert und auch das Duo- 
denum größtenteils noch im Abdomen vorhanden war, mußte er von den vorgefallenen Teilen die tiefste Lage im linken Pleuraraum einnehmen. Über und hinter ihm bemerkt man das Darmkonvolut, das die zweilappige linke Lunge stark nach oben verdrängt und etwas verdreht hatte. Das Herz war nach rechts verdrängt, die rechte Lunge gewöhnlich gebildet.

Fall VI. (Mus.-Kat. Nr. 5477.) Leiche eines 7 Tage alten, in der Hebammenschule des Herrn Prof Dr. Freund geborenen, am 13. Mai 1908 sezierten Mädchens. Der Körper $53 \mathrm{~cm}$ lang, $3110 \mathrm{~g}$ schwer, ohne äußerlich wahmehmbare Mißbildung. Die Lippen stark zyanotisch, die weichen Schädeldecken ziemlich blutreich. Der Schädel miBt im Horizontalumfang $33 \mathrm{~cm}$. Bei Eröffnung des Rumpfes zeigt sich ein fast für zwei Finger durchgängiger Defekt in der

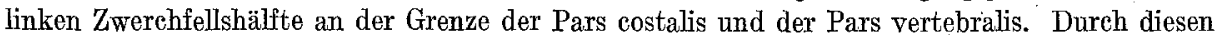
Defekt ist der Magen samt dem Netz und samt dem mit einem freien Gekröse versehenen Darm bis zur Flexura coli sinistra, ebenso wie die Milz in die Pleurahöhle durchgetreten. Im Zusammenhang damit ist die linke Lunge hypoplastiseh, das Herz samt dem Herzbeutel sehr stark nach rechts disloziert und die rẻchte Lunge partiell atelektatisch. Die Milz ist von gewöhnlicher Größe and liegt rückwärts medial am hinteren Mediastinum nahe der Aorta (Abb. 11). Die Zwerchfells-

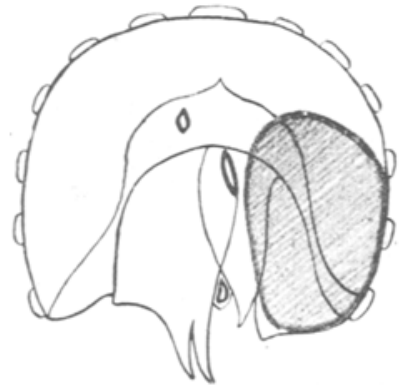

Fig. 10. Mus.-Kat. Nr. 4452.

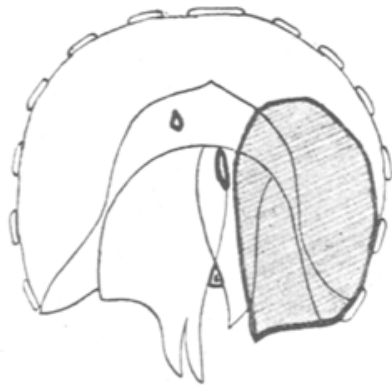

Fig. 11. Mus.-Kat. Nr. 5477.

lücke nimmt mehr als die Hälfte des linken Diaphragmateiles ein. Vorne und seitlich reichte der Defekt nicht bis an die Brustwand, sondern wird durch eine sichelförmige Zwerchfellsfalte, die sich allerdings nur sehr schmal bis über die 10. Rippe hinaus erstreckt, eingesäumt, während hinten als Grenze des Defektes die Serosa der Leibeshöhlenwandung wahrgenommen wird.

Fall VII. (Mus.-Kat. Nr. 6885.) $29 \mathrm{~cm}$ langer, weiblicher Fötus mit vollständiger Enzephalo-Myeloschisis, der dem Institute von Herrn Dr. Will in Diemerdingen am 4. Jannar 1910 eingesandt worden war. Die Sektion der Rumpforgane ergab nur eine Hypoplasie der Nebennieren, welche kaum $1 / 4 \mathrm{ccm}$ groß waren. Weiterhin fand sich noch ein für zwei Finger durchgängiger Defekt in der linken Zwerchfellshälfte, durch den ein Teil des linken Lappens der vergrößerten Leber, die Milz, der Magen und einzelne Dünndarmschlingen in die linke Pleurahöhle durchgetreten waren. Durch die sehr hoch, weit nach hinten und medial in den Brustraum verlagerte Milz war das hintere Mediastinum nach rechts ausgebaucht worden, wie auch das Herz stark nach rechts verdrängt erschien. Das in der Bauchhöhle vorhandene Duodenum war knapp hinter der Einmündung des Ductus choledochus steil nach oben umgeknickt, der Magen lag ebenfalls zum gröBten Teil in der Brusthöhle, unmittelbar vor der Milz, bedeckt von den vorgefallenen Darmschlingen, die dem Jejunum, dem Ileum, dem Colon ascendens und dem Colon transversum angehörten. Das Colon besaß ein freies Gekröse, das unmittelbar in jenes des Ileum überging. Was die Zwerchfellslücke betrifft (Abb. 12), so fehlt die linke Hälfte des Diaphragma fast vollkommen, nur eine schmale und kurze, bis zur 8. Rippe reichende Zwerchfellsichel begrenzt nach vorne und etwas lateral den Defekt, während er im übrigen an die Serosa der Leibeshöhlenwand angrenzt. 
Fall VIII. (Mus.-Kat. Nr. 6942). $46 \mathrm{~cm}$ langer totgeborener Knabe von der Klinik des Herrn Geheimrat Prof. Dr. Fehling. Sektion am 14. Oktober 1910. Äußerlich war an der Leiche eine Mißbildung nicht wahrzunehmen, doch ergab die Sektion einen Truncus arteriosus communis persistens, sowie eine große Defektbildung in der linken Zwerchfellshälfte, die sich nahezu völlig über die Pars costalis und lumbalis erstreckte (Abb. 13), sodaB vorne der Rand des Defektes bis auf einen Zentimenter an das Perikard heranreichte, obwohl das Herz mit dem Mediastinum stark nach rechts verdrängt war. Nur medial grenzte der Defekt an einen schmalen Muskelstreifen, der neben dem Ösophagus vorbei nach hinten gegen die Wirbelsäule hinzog. Durch den Defekt ist der linke Leberlappen vollständig in die Pleurahöhle vorgefallen, eine tiefe Einschnürung grenzt ihn gegenüber dem rechten Lappen der vergröBerten Leber ab. Diese Schnürfurche befindet sich an jener Stelle, wo der Rand des Zwerchfelldefektes über die Leber hinwegläuft. Nach dem Herausziehen dieses Leberlappens aus der Pleurahöhle erscheint die sehr rudimentäre linke Lunge, die nur einen etwa $2 \mathrm{~cm}$ messenden Komplex darstellt. Unterhalb derselben ist eine hernienartige Nische des linken Pleuraraumes, der einen spaltartigen Zugang zwischen Aorta und Ösophagus besitzt und hinter dem Herzen gelegen ist. In dieser Nische ist die gewöhn-

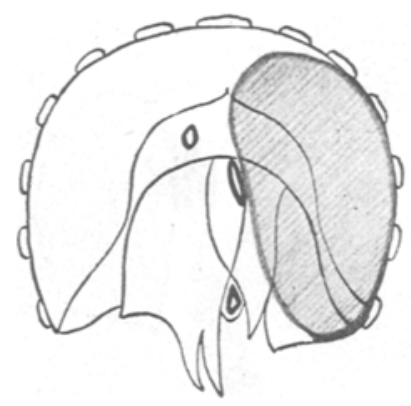

Fig. 12. Mus.-Kat. Nr. 6885.

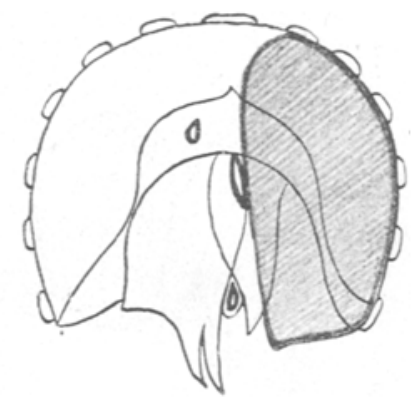

Fig. 13. Mus.-Kat. Nr. 6942.

lich große Milz eingelagert, aber nirgends mit der Wand verwachsen. Auch der Magen ist mit seinem Fundusteil in die Pleurahöhle disloziert, der Nervus phrenicus verläuft in der medialen Wand der Pleurahöle und verzweigt sich auf der pleuralen Fläche des Restes der linken Zwerchfellshälfte in der gewohnten Weise. Die rechte Lunge ist zweilappig, der Dickdarm besitzt ein freies Gekröse, das allerdings im Bereiche des Colon transversum sehr kurz ist. Zökum, Colon ascendens, Colon transversum und eine große Zahl von Dünndarmschlingen sind ebenfalls vorgefallen. Das Colon descendens zeigt jedoch kein freies Gekröse, es ist wie gewöhnlich angewachsen. Dagegen erweist sich das S romanum auffallend lang und mit ausgedehntem Aufhängeband versehen, so daß es sich in dreifacher Schlingenanordnung winden mußte. Der Pylorus wird unterhalb des Zwerchfells bemerkt, ebenso wie das Duodenum. Am Magenfundus ist das Netz und ein Konvolut von Dünndarmschlingen angewachsen, ebenso wie die aus ihrem Bett herausgetretene und stark nach links und oben gezerrte Gallenblase mit der vorderen Magenwand verwachsen erscheint. Diese Zerrung der Gallenblase nach links und oben wurde offenbar durch den Eintritt des linken Leberlappens in die linke Pleurahöhle bedingt, wobei auch der verbreiterte rechte Leberlappen in Lage und Form eine Veränderung erfuhr und sich etwas nach links hin dehnte. Zwischen der Unterfläche der Leber und der Vorderfläche des Magens bildete sich eine ungewöhnlich breite Bindegewebsbrücke, in der auch der Fundus der Gallenblase sein Lager fand. Das Foramen Winslowi war vollkommen durch Bindegewebshüllen verschlossen.

Kurz rekapituliert ergeben diese 12 Fälle von Zwerchfellsdefekt übereinstimmend eine Lückenbildung im dorsalen Diaphragmateile; auch die 
rechtsseitigen Fälle III und IV gehören hierher, worauf unten ausführlicher einzugehen ist. Was die Größe des Defektes anlangt, so zeigen die linksseitigen Fälle I bis VIII einen zunehmenden Umfang der Lückenbildung auf Rechmung fast der ganzen kostalen und lumbalen Zwerchfellspartie, ähnlich, wie dies v. Gößnitz seinerzeit zeigen konnte (A).

Eine auffallende Übereinstimmung zeigt sich in dem Verhalten der dem Zwerchfell benachbarten, meistens prolabierten Organe; in den linksseitigen Fällen sowie in dem rechtsseitigen Fall II war ein freies Gekröse des Kolons konstatierbar, fast stets erwies sich die Leber als sehr grob, oder auch als deutlich vergrößert, nie aber als verkleinert; die linksseitigen Fälle zeigten ferner eine eigenartige im ganzen und großen stets wiederholte Anordnung der in die linke Pleurahöhle vorgefallenen Eingeweide, insofern stets die Milz am weitesten dorsal und medial lag, nach vorne vom Magen und nach oben von Darmschlingen verdeckt; in der spaltartigen" bis nischenförmigen Ausbuchtung des hinteren Mediastinums nach rechts hin zu einer Art von „Pleuranebensack", wie ihn Wenzel Gruber in einem analogen Falle gesehen und abgebildet hat, sowie in der gleichsinnigen Verdrängung des Herzens nach rechts stimmen ebenfalls die Fälle der linken Seite auffallend überein. Ganz analog zeigt der rechtsseitige Fall II eine Mediastinalausbauchung nach links hin. Nur der linksseitige Fall $V$ steht insofern allein, als er durch. eine Nebenlungenbildung kompliziert ist, ein Verhalten, das bisher nur in wenigen Fällen von Zwerchfellsmißbildung festgestellt werden konnte (Kohn, Kaup, Robsmann, Beneke).

Über die Entwieklung der dorsal gelegenen kongenitalen Zwerchfellsdefekte ist viel geschrieben worden, und ich darf unter Hinweis auf die jüngsten Ausführungen Caillouds, die dureh meinen rechtsseitigen Fall III veranlaßt worden sind, mich wohl kurz fassen. Der angeborene dorsale Zwerchfellsdefekt entsteht infolge einer Hemmung in der gehörigen Ausbildung der Plica pleuroperitonaealis, bzw. in einem Zurückbleiben oder in einem Mangel des hinteren Uskowschen Pfeilers; dadurch wird es unmöglich, daß das häutige Zwerchfell der betreffenden Seite zum Verschluß gelangt. Von der Ausbildung der Muskulatur des Diaphragma hängt dagegen eine solche Zwerchfellslüekenbildung nicht $a b$; infolgedessen ist auch der oft gelesene Satz, daß der dorsal gelegene Zwerchfellsdefekt zum Foramen Bochdaleki (= lumbocostale) genetisch in Beziehung stünde, nicht richtig. Durch ungenügende Ausbildung oder gänzlichen Mangel des muskulären Gewebsanteiles im Zwerchfell wird aber wohl, vorausgesetzt, daß sich das seröse Zwerchfell gebildet hat - die Möglichkeit zur Entstehung einer Zwerchfellshernie gegeben. Unter Zwerchfellshernie ist jedoch im Anschluß an die einzig zulässige und z. B. auch von Treitz gegebene Definition des Begriffes „Hernie" nur eine sackartige Vorstülpung und Dehnung einer Zwerchfellspartie mit einer eventuell mehr oder minder großen Eingeweideverlagerung innerhalb des „Bruchsackes" zu verstehen, also jene Bildung, die in der Literatur unter dem 
Namen einer "Hernia diaphragmatica vera" bekannt ist. Der Ausdruck "Hernia falsa oder spuria" für eine wirkliche Zwerchfellslückenbildung ist ungenau und irrefuhrend; er sollte daher aufgegeben werden.

Wir verdanken W. v. Gößnitz (A u. B) eine recht eingehende Darstellung der kongenitalen Lückenbildung des Diaphragma, ganz besonders der Verhältnisse an den Nervi phrenici und an der Zwerchfellsmuskulatur; immerhin hat aber dieser Autor den Untersehied zwisehen Hernienbildung und Lüekenbildung in seinem letzt beschriebenen Falle (B) nicht gewahrt, da er von ihm sagt, der Fall gehöre ,seiner morphologischen Stellung nach zu jener Gruppe von falschen Zwerchfellshernien, die durch Entwicklungshemmung der Muskulatur.einer Seite entstanden sind". Gewiß war in seinem Falle die völlige Ausbildung der Muskulatur gehemmt, sie war es aber, weil sich das Foramen pleuroperitaoneale der linken Seite nicht geschlossen, weil sich mit anderen Worten das membranöse Zwerchfall nicht gebildet hatte. Meines Erachtens setzt W. v. Gößnitz den teratogenetischen Terminationspunkt für die Genese der kongenitalen Zwerchfellslücke zu spät an, wenn er die Ansicht vertritt, daß eine Wachstumshemmung der Muskulatur das Offenbleiben des Foramen pleuroperitonaeale bewirkt. Gewisse Beobachtungen an eigenen Fällen dürften meine Ansicht von einer früheren Terminationsperiode der Zwerchfellslückenbildung stützen.

Wie dies für die rechtsseitigen Fälle I u. II, sowie für den linksseitigen Fall II angeführt werden konnte, kommt es vor, daß der Zwerchfellsdefekt nicht sensu strictiori von der glatten hinteren Körperwand allein begrenzt wird, sondern daß sich ein allerdings minimal ausgebildeter, schmaler und flacher, dünner Muskelstreifen unter der Serosa an der Grenze zwischen Brust- und Bauchhöhle von der Gegend des Hiatus oesophageus her zur 12. bis 11. Rippe hinzieht, ein Muskelstreifen, der so dünn ist, daß gleichwohl am Rande des Defektbereiches die Serosa der Bauchhöhle ohne die geringste faltenartige Vorwölbung in die Serosa der Brusthöhle übergehen kann. Gleichwohl ist in solchen Fällen der Zwerchfellsdefekt völlig innerhalb eines muskulären Randes gelegen, denn auf der anderen Seite reicht die muskelhaltige kostale Zwerchfellssichel bis zur 11. u. 12. Rippe zurück, ja hier stoßen die beiden Muskelfaserzüge so stumpfwinklig aufeinander, daß es aussieht, als ob sie sich ineinander fortsetzen würden, und zwar an der Stelle, wo sonst das Foramen lumbocostale (= Bochdaleki) zu suchen ist. Wie schon im Anschluß an den I. rechtsseitigen Fall ausgeführt ist, kann dieses Verhalten der lateralen Lumbalportion dem Bogenbündel des Albinus beim Erwachsenen entsprechen. Es ist nun kaum anzunehmen, daß diese lumbale Muskelportion doch die enge Straße an der hinteren Begrenzung des Foramen pleuroperitonaeale in diesen seltenen Fällen durchwachsen hätte, wenn tatsächlich die Ursache des Offenbleibens des Foramens in einem. Mangel an Wachstumsenergie des Zwerehfellsmuskels gelegen wäre. Es scheint mir vielmehr im allgemeinen so zu sein, daß die wachstumskräftige Muskulatur an der Weiterentwicklung gehemmt wird, 
weil ihr durch eine Lückenbildung im primär entstandenen membranösen Zwerchfell nicht genügend Raum geblieben ist, und da $B_{i}$ in speziellen Fällen in einem trotz dieses Hindernisses erfolgten Einwachsen von auch nur rudimentären Muskelzügen mehr die Randserosa oder in einer wulstartigen Muskelverdickung im Randbereiche des offenen Pleuroperitonäallochs die Wachstumstendenz des muskulären Zwerchfelles gut zum Ausdruck kommt. Für diese Überlegungen ist zunächst ganz g'eichgültig; ob die Genese der Zwerchfellslücke auf einem primär nicht zustande gekommenen Verschluß der Pleuroperitonäalmembran oder auf einem Wiederdurchreißen derselben durch sehr frühzeitiges intrauterines Trauma mit Prolaps von Baucheingeweiden bedingt ist, wie dies von manchen Autoren angenommen wurde.

Die Beobachtung der phrenischen Muskeleinwachsung unter die nicht faltenartig abgehobene Serosa der hinteren Körperwand gibt Anlaß, auf meinen rechtsseitigen Fall III (Fall von Cailloud) und auf den rechtsseitigen Fall IV hinzuweisen, zu welchen der Fall IV von W. v. Gößnitz (A) in Parallele steht. Hier handelt es sich um Lückenbildungen zwar auch im dorsalen Zwerchfellsanteil, die aber nicht unmittelbar an der Körperwandung gelegen, sondern von ihr abgerückt sind, so daß allseitig eine ringförmige Zwerchfellsfalte den Defekt umgrenzt. Man muß für solche Fälle, welche die Franzosen (Duguet) einem Knopfloch verglichen haben, - zumal wenn sie erwachsene Individuen betreffen - die traumatische Genese zunächst sicher ausschalten. In meinen Fällen scheint dies in genügendem Maße möglich zu sein, da keinerlei Adhäsionen zwischen dem Rand der Zwerchfellslücke und den vorgefallenen Organen bestehen. Wie muß man sich die Morphogenese solcher Lückenbildungen vorstellen? Betrachten wir ihren Rand noch einmal genau, so sehen wir, daß eine recht ansehnlich ausgebildete, der anderen Zwerchfellshälfte kaum nachstehende Muskulatur den Defekt einschließt und, daß diese Muskulatur im ehemaligen Bereiche der 11. bis 12. Rippe eine mehr oder weniger winkelige Faserüberschneidung aufweist, wie dies in der Gegend eines gut ausgebildeten Foramen lumbocostale (= Bochdaleki) der Fall zu sein pflegt. Cailloud hat die Möglichkeiten des Zustandekommens dieser Lückenanordnung bereits dargestellt; sie sei hier kurz wiederholt. Es ist nicht unbedingt nötig, daß das Foramen pleuroperitonaale (etwa in Ermangelung eines dorsalen Uskowsehen Pfeilers) bei der Weiterentwicklung absolut dorsal an der Körperwand gelegen sein muß. Wenn dieser Pfeiler angelegt wird, aber im Wachstum zu bestimmter Zeit zurückbleibt, dürfte das Pleuroperitonäalloch durch eine mehr oder minder von der Dorsalseite vordrängende Falte von der Leibeshöhlenwand abgedrängt sein. In diese Serosafalte vermag Muskulatur einzuwachsen und in deutlicher Weise die lumbalen Schenkel auszubilden. Sahen wir doch sogar in den erwähnten drei Fällen diese Muskulatur unter der Serosa allerdings in etwas variierter Form einwachsen, ohne daB solch eine Falte angedeutet war. Und gerade hierin scheint mir ein Hinweis auf die Möglichkeit zu liegen, daß eventuell bei längerem Leben und bei der 
fortgesetzten Atemfunktion es zu einer noch kräftigeren Ausbildung dieser auch vom N. phrenicus versorgten Muskelpartie in der hinteren Körperwand kommen kann, und daß diese Muskelpartie sich allmählich von der Körperwand unter Vordrängung der Serosa gegen den Defekt wulstig oder faltenartig abhebt und sich beim Wachstum des Thorax mehr und mehr von der Körperwand emanzipiert, so daß die ebenfalls von der dorsalen Körperwand abrïckende Zwerchfellslücke eine nunmehr ringförmige Zwerchfellsleiste zur Begrenzung erhält. Der Vorgang der Abdrängung von der Körperwand spielt schon intrauterin bei der membranösen Zwerchfellsbildung eine Rolle und gewiß auch bei der mehr zentral gelegenen angeborenen Zwerchfellslückenbildung, wenn nur ein hinterer Schenkel der Plica pleuroperitonaealis angedeutet wạr. Ist natürlich weder solch ein Schenkel der Serosafalte angedeutet gewesen, noch eine Einwachsung von phrenischer Muskulatur unter die lumbodorsale Serosawand zustande gekommen, dann wird wohl keinerlei Möglichkeit ersichtlich sein, daß der Zwerchfellsdefekt im späteren, extrauterinen Leben eine Verschiebung nach den Centrum tendineum hin erfährt.

Die kausale Genese der Zwerchfellslücken ist recht unklar. W. v. Göbnitz (B) hat folgende Ätiologiemöglichkeiten aufgestellt: 1. Fötalerkrankungen und Lageanomalien der Kinder. 2. Erkrankungen und Traumen der Mutter in entsprechenden Schwangerschaftswochen.

Was die Fötalerkrankungen anbelangt, so sind sie für das Zustandekommen der Zwerchfellsdefekte und Zwerchfellshernien vorläufig durchaus unbewiesen - wenn man nicht Mißbildungen der Kinder iberhaupt auch als Fötalerkrankungen bezeichnen will. Hemmungen im Zwerchfellsschluß kommen als alleinige Mißbildung offenbar aus immanenter, autonomer Ursache vor, ebenso als Mißbildung in Kombination mit anderen (Myeloschisis, Anenzephalie, Herz- und Gefäßmißbildungen, Skelettanomalien usw.), wobei in seltenen Fällen die Zwerchfellsmißbildung durch andere Mißbildungen der Körperform z. B. bedingt sein kann. Das durch Erkrankung oder Mißbildung des Zentralnervensystems unterbliebene oder verzögerte Auswachsen der phrenischen Nervenwurzeln ist für ein Offenbleiben der Foramina pleuroperitonaealia nicht maßgebend. Dadurch wird nur die Bildung des muskulären Anteils am Zwerchfell gestört. Was die Lageanomalien der kindlichen Frucht anbelangt, so wies bereits Cailloud auf die geringe Wahrscheinlichkeit der Annahme hin, daß sie bei der frühzeitigen teratogenetischen Terminationsperiode der kongenitalen Zwerchfellslücken in Betracht kommen. „Traumen der Mutter in entsprechenden Schwangerschaftswochen" scheinen mir auch als Ursache für die in Rede stehenden Bildungen sehr fraglich zu sein. Der Hinweis auf die Wirkung, welche eine nicht einmal besonders heftige, eventuell fortgesetzte Erschütterung auf die Entwicklungsfähigkeit unbebrüteter Eier bzw. auf die regelrechte Entwicklung befruchteter Eier verschiedener Anamnier hat, genügt meines Erachtens nicht für den Nachweis, daß durch Erschütterung in den ersten Sehwangerschaftswochen der Grund zu solcher Mißbildung gelegt wird, zumal doch wohl manche Frauen trotz nicht 
geringer traumatiseher Einwirkung in der frühen Schwangersehaft, oder trotz fortgesetzter, anstrengender körperlicher Arbeit und der damit verbundenen Unbilden (landwirtschaftliche Arbeit!) wohlentwickelte Kinder geboren haben. Zwar muß man die Möglichkeit einer solchen traumatischen Ursache zugeben (F. Mar chand), aber mehr als eine mutmaßliche Möglichkeit dieser Ursache für die in Frage kommende Zwerchfellsmilbildung wird aus dem bisher in der Literatur niedergelegten Beobachtungs- und Erfahrungsschatz nicht erkannt werden können. Für die kongenitalen Zwerchfellslïeken kann man mit Marchand wohl am ehesten auf eine lokale Entwicklungshemmung rekurrieren. Die Häufigkeit und Gleichartigkeit dieser Bildung auf der einen Seite, anderseits die fast stets gänzlich im Stiche lassenden Schwangerschaftsanamnesen der Mütter sprechen hier nicht für eine traumatische Ursache, wenn auch im letzten speziellen Falle von W. v. Gößnitz ein traumatisches Ereignis während der frühen Schwangersehaftszeit angegeben werden konnte.

Man hat auch daran gedacht, daß Entwicklungsstörungen der Leber, des Gekröses, des Magens, zu geringe Ausbildung der Lungen an der Persistenz des Zwerchfelloches schuld sein könnten. Alle diese Momente sind lebhaftest diskutiert, angenommen, verworfen und wieder angenommen worden. Tatsächlich weiß man nichts Bestimmtes darüber. So soll einerseits die sich mächtig entwickelnde Leber ${ }^{1}$ ) imstande sein, den Pleuroperitonäalverschluß zu beschleunigen, ein Argument, das in Rücksicht auf den größeren rechten Leberlappen für den frühzeitigen Zwerchfellsverschluß und das seltenere Vorkommen der Zwerchfellsmißbildungen auf der rechten Seite angeführt wird, andererseits soll die zu große Leber am Zwerchfellsdefekt schuld sein. Manche Autoren haben im Gegensatz dazu, aber ebenfalls ohne Anklang und Bestätigung zu finden, zu geringe Leberausbildung als Ursache des verzögerten Zwerchfellsverschlusses bezeichnet. Daß bei dem Vorhandensein eines freien Gekröses leichter Darmteile durch das Zwerchfelloch prolabieren, muß als selbstverständlich angesehen werden, kann aber nicht als ätiologisehe Erklärung für die Zwerehfellsdefekte gelten, zumal es ja auch kongenitale Zwerchfellsdefekte ohne Mesenterium commune gibt. Vielmehr scheint die Hemmung in der Fertigbildung bzw. Verklebung der Mesenterialserosa etwas Analoges zu der Hemmung im Verschluß der Zwerchfellserosa darzustellen; wie E. Schwalbe ausgeführt hat, macht sich diese Hemmung ferner darin bemerkbar, då ganz regelmäßig das freie vorgefallene Gekröse auch in der Pleurahöhle keine Verlötung eingeht. Die Aplasie, besser Hypoplasie der Lungen, die W. v. Gößnitz in Frage zieht als eine Ursache des Zwerchfellsdefektes, kann bestimmt nicht eine solche ätiologische Bedeutung haben. Vielmehr dürfte durch den Prolaps der Baucheingeweide bei offengebliebenem Foramen pleuroperitonaeale die Lunge verdrängt und verkümmert sein. Wissen wir ja doch aus den oben er-

1) Nach E. Schwalbe kann man die so häufig bei kongenit. Zwerchfellsmißbildrangen gesehene Lebervergrößerung auch als eine Form von Hemmungsbildung (Hemmung der physiologischen Rückbildung auf das Normalgrößenverhältnis) ansehen.

Virchows Archiv f. pathol. Ant. Bd. 218. Hft. 1. 
wähnten Fällen Kohns, Kaups, Robsmanns bzw. Benekes, denen sich mein linksseitiger Fall $\mathrm{V}$ anschließt, daß eben die Umstände, die beim Zwerchfellsdefekt eine Rolle spielen, gleichzeitig wohl eine Defektbildung der Lunge bedingen können durch Abschnürung eines Teiles der embryonal weit ins Foramen pleuroperitoneale vorragenden Lungenanlage.

Die Bevorzugung der linken Seite durch Zwerchfellslücken und auch durch Zwerchfellshernien ist offenkundig, wurde aber doch anscheinend überschätzt. Cailloud fand durch sorgfältigstes Nachprüfen in der Literatur die Zahl des rechtsseitigen Defektes bedeutend größer als dies die neuesten Zusammenstellungen angegeben haben, aber immerhin beläuft sich die Kasuistik der linksseitig̀en Defekte auf eine vier- bis fünfmal höhere Zahl. Diese Bevorzugung der linken Seite scheint, wie Caillo u d ebenfalls im Anschluß an eine Reihe von Autoren angenommen, im normalen Entwicklungsvorgang seinen Grund zu haben, der das Foramen pleuroperitonaeale dextrum sich rascher schließen läßt, als das linke. Gewiß muß aber auch anerkannt werden, daß die große Leber auf der rechten Seite, der mobile Magen und Dünndarm auf der linken die Verhältnisse ungünstiger für den normalen Schluß der linken Zwerchfellshälfte gestalten, ohne damit die Ansicht von der ausschließlichen Schutzwirkung der Leber für den Schluß des rechten Foramen pleuroperitonaeale unterschreiben zu wollen. Denn mitunter bleibt dies Foramen trotz gut entwickelter Leber offen, und dann sieht man, wie sich die Leber in der Form den ungewöhnlichen Raumverhältnissen anpaßt, indem sie entweder wie ein Knopf in die Öffnung einwächst, oder aber indem sie selbst nach der rechten Pleurahöhle unter stärkster Modifikation ihrer Gestalt, also bei anscheinend völlig ungestörter, eigener Funktion durchtritt. Gerade für diese Punkte ist mein rechtsseitiger Fall III (vgl. Cailloud) ein guter Hinweis. Überhaupt sind ja, wie zahlreiche Fälle lehren, größere Zwerchfellshïcken mit Vorfall ausgedehnter abdomineller Eingeweideabschnitte durchaus nicht absolut lebensschädlich (vgl. Thoma). Das wird wohl vor allem auch bedingt durch die schon erwähnte und von den Autoren wiederholt betonte Beobachtung, dab die in die Brusthöhle prolabierten Darmabschnitte und Mesenterialteile dort primär nicht zur Verklebung der Serosen neigen. Im Gegensatz zu den größeren Defektbildungen des Zwerchfells sind die kleinen gefährlicher, da sie leichter zur Darmeinklemmung Anlaß geben, wie sie sich auch bei dem letzt beschriebenen Falle W. v. Gößnitz' (B) (3 Jahre altes Mädchen) ereignet hatte.

Jedenfalls dürfte eines aus diesen Ausführungen hervorgehen, nämlich daß das Thema der Zwerchfellslücken- und Zwerchfellshernienbildung noch genug ungelöste Fragen darbietet, gewiß mehr, als daß man nicht an der Hand geeigneten Materiales zur Klärung des einen und anderen Punktes beitragen sollte. 


\section{Literatur.}

Beneke, Über Bauchlunge und Hernia diaphragmatica spuria. Verh. d. D. Path. Ges., Bd. 9, S. 202, 1905. - Cailloud, Über einen rechtsseitigen Fall von Zwerchfellsdefekt beim Erwachsenen. Virchows Arch., vorstehende Abhandlung. - Ehrmann, Nouveau catalogue du musée d'anatomie normale et pathologique de la facultée de médecine de Strasbourg 1843. - Eisler, Die Muskeln des Stammes. In Bardelebens Handbuch d. Anat. Jena 1912. v. Gößnitz, [A], Sechs Fälle von linksseitigem Zwerchfellsdefekt. Jenaische Ztschr. f. Naturwissensch., Bd. 38, S. 618, 1903. - v. GöBnitz, [B], Ein weiterer Beitrag zur Morphologie des Zwerchfells. Ebenda Bd. 39, S. 235, 1905. - Gruber, G. B., Über Nebenlungenbildung bei kongenitalem Zwerchfellsdefekt. Zieglers Beitr. z. path. Anat. u. z. allg. Path. 1914. - Gruber, Wenzel, Abhandlung eines Falles mit einem Mesenterium commune usw. Virchows Arch. Bd. 47, S. 382, 1869. - Joest, Bemerkungen zu der Arbeit von Stöber. Frankf. Ztschr. f. Path. Bd. 9, S. 478, 1912. - Kaup, Zwei Fälle von Hernia diaphragmatica congenita mit Abtrennung eines Lungenteiles. I.-D. Kiel 1891. - Kohn, Über Hernia diaphragmatica congenita. I.-D. Erlangen 1874. - Marchand, F., Mißbildungen in Eulenburgs Realenzyklopädie, Bd. 9, 1910, (4. Aufl.) S. 729 u. 824. - Robsmann, Über retroperitonäale Zysten der Bauchhöhle. I.-D. Königsberg 1904. - Schwalbe, E., Über einen Fall von linksseitigem angebor. Zwerchfellsdefekt." Morpholog. Arbeiten Bd. 8, S. 135. - Derselbe, Die Morphologie der MiBbildungen Bd. 1, S. 174, 1906. - Derselbe, țber kongenitale Zwerchfellshernien. Münch. med. Wchr. 1899, S. 12. - Stoeber, Eine Hernia diaphragmatico-peritonaealis bei einem Hunde. Frankf. Ztschr. f. Path. Bd. 10, 1912. - Treitz, Hernia retroperitonaealis. Prag 1875. Winkler, Angeborene Zwerchfellshernie mit bemerkenswerten Mesenterialverhältnissen. Frankf. Ztschr. f. Path., Bd. 6, S. 370, 1911. - (Weitere Literatur siehe in der Tabelle der rechtsseitigen angeborenen Zwerchfellstï̈cken und dem Literaturverzeichnis von Cailloud.)

\section{VII. \\ Die besondere Form der durch Nikotin verursachten Aorten- veränderung.}

(Aus dem Pathologisehen Institut des Augusta-Hospitals zu Berlin.)

Von

Dr. C. Rehr.

Ätiologie und Genese der Arteriosklerose sind noch nicht völlig klargestelit, allerdings wird immer mehr (vgl. Thoma, Marchand, Romberg) die Ansicht vorherrschend, dab die Arteriosklerose im wesentlichen die Folge abnorm gesteigerter funktioneller Inanspruchnahme der Arterien ist, d. h. also durch mechanische Einwirkungen verursacht wird.

Für diese Annahme spricht die Tatsache, daß die Erkrankung mit dem Alter an Häufigkeit zunimmt, daß dieselbe in erster Linie an denjenigen Stellen der Gefäßwand auftritt, wo sich Abgangsstellen kleinerer Gefäße finden, die Reibung eine größere, der Druck ein höherer ist. Auch die Feststellung, daß die Arteriosklerose häufiger bei muskulösen stark arbeitenden Männern als bei Frauen gefunden wird und daß manche mit schwerer Arbeit verbundene Berufsarten besonders betroffen sind, wird als beweisend für jene Annahme erachtet: hierbei herrscht infolge außergewöhnlich lebhafter Herzaktion eine höhere Spannung im Arteriensystem. 